\title{
DIFERENCIAS SOCIOTERRITORIALES DEL ESTADO DE GUERRERO, MÉXICO: UNA PROPUESTA DE ANÁLISIS GEOGRÁFICO-ECONÓMICO
}

\author{
SOCIOTERRITORIAL DIFFERENCES OF THE STATE OF GUERRERO, MEXICO: \\ A PROPOSAL OF GEOGRAPHIC-ECONOMIC ANALYSIS
}

\author{
Neftalí García Castro', Yuridia Lozano Peñaloza² \\ 1 Universidad Autónoma de Guerrero (UAGRO), Chilpancingo, México \\ 2 Universidad Autónoma de Guerrero (UAGRO), Acapulco, México
}

Correspondência para: Neftalí García Castro (ngarciaca@conacyt.mx)

doi: 10.12957/geouerj.2019.44833

Recebido em: 27 ago. 2019 | Aceito em: 17 set. 2019

\section{RESUMEN}

Este artículo presenta las diferencias territoriales que existen en el estado de Guerrero, México. Se retoman algunos lineamientos conceptuales y metodológicos expuestos por especialistas formados en el Instituto de Geografía de la Universidad Nacional Autónoma de México (UNAM). La investigación es resultado de la consulta de fuentes documentales y estadísticas, las más recientes posibles. En algunos casos, la falta de datos actualizados se subsano con la observación directa en campo. En la primera parte del trabajo se exponen aportes cognoscitivos fundamentales que han dado pauta para medir las particularidades geográfico-económicas del territorio guerrerense, en investigaciones geográficas previas sobre la entidad. Posteriormente, se explica el método del Valor índice Medio (VIM) como procedimiento estadístico para examinar integralmente el comportamiento de los indicadores que se compendian, de acuerdo al marco de referencia de esta investigación. Y se revela una tipología que clasifica a las jurisdicciones del estado de Guerrero según sus particularidades socio-territoriales. En ese orden de ideas, uno de los principales hallazgos gravita en torno al hecho de que algunas de las circunscripciones guerrerenses con condiciones socioeconómicas por debajo de la media estatal no siempre se asocian con aquellos espacios marginales y cuyas dificultades sociales han sido una constante a lo largo de la historia económicoproductiva de la entidad.

Palabras-clave: diferencias territoriales. valor índice medio. asimilación económica. enfoque geográfico.

\begin{abstract}
This article presents the territorial differences that exist in the state of Guerrero. Some conceptual and methodological guidelines presented by specialists trained in the Institute of Geography of the National Autonomous University of Mexico are retaken. The research is the result of consulting documentary sources and statistics, the most recent possible. In some cases, the lack of updated data was corrected with direct observation in the field. In the first part of the work are exposed fundamental cognitive contributions that have given pattern to expose the geographic-economic peculiarities of the Guerrero territory, in previous geographical investigations on the entity. Subsequently, the method of the Mean Index Value (VIM) is explained as a statistical procedure to comprehensively examine the behavior of the indicators that were selected, according to the frame of reference of this research. And it reveals a typology that classifies the jurisdictions of the state of Guerrero according to their socio-territorial particularities. In that order of ideas, one of the main findings gravitates around the fact that many of the Guerrero districts with socioeconomic conditions below the state average are not always associated with those marginal spaces and whose social difficulties have been a constant throughout the economic-productive history of the entity.
\end{abstract}

Keywords: territorial differences, average index value, economic assimilation, geographic focus.

\section{INTRODUCCIÓN}


Este artículo presenta las diferencias territoriales que existen en el estado de Guerrero. Se retoman algunos lineamientos conceptuales y metodológicos expuestos por especialistas formados en el Instituto de Geografía de la Universidad Nacional Autónoma de México. En ese orden de ideas, destacan los aportes de Propin y Sánchez (1998), Vázquez, (2000), así como García et al. (2016), ya que sus investigaciones han gravitado en torno a las particularidades geográfico-económicas del territorio guerrerense. Es importante mencionar que Propin y Sánchez han impulsado la Teoría de la Asimilación Económica del Territorio para coadyuvar a la interpretación de la relación que guardan ciertos componentes sociales, económicos y físicos, en un espacio particular (García, 2011). En la literatura especializada se señala que la Asimilación Económica debe ser entendida como la incorporación gradual de distintos espacios a la vida económica del territorio al que pertenecen (García, 1993).

En el caso de los trabajos anteriores sobre el estado de Guerrero, se ha optado por la Tipificación Probabilística como solución metodológica propuesta por la Teoría de la Asimilación Económica del Territorio; esta herramienta estadística ha dado pauta para revelar agrupamientos de procesos u objetos de interés, a partir de la repetividad, similitud, homogeneidad y jerarquía territorial (Propin, 2003). El enfoque tipológico resume distintos atributos asociados con el hecho, fenómeno o proceso en cuestión; lo que facilita la conformación de grupos o tipos discretos que, en lo fundamental, constituyen instrumentos valiosos para examinar integralmente el comportamiento de las variables o indicadores que se compendian, de acuerdo al marco teórico-conceptual de referencia (Sánchez, 2000).

En el caso de la presente investigación se plantea como alternativa metodológica al Valor Índice Medio (VIM), la secuencia asociada con éste es sencilla y permite obtener resultados similares a los conseguidos mediante la Tipificación Probabilística o el método de Componentes Principales, ya que, por ejemplo, este último "depende de la correlación entre los indicadores, de manera tal que a mayor asociación el índice final resultará más representativo. En contraste, de tener una relación mediana o baja, el índice reflejará en menor medida los parámetros originales. Aquí se presenta una especie de paradoja: si las variables a cuantificar tienen alta correlación entre sí entonces meramente repiten la información original. Y si se eliminan los elementos con mayor interrelación entonces el índice global tendrá una 
representatividad muy pobre”. (García de León, 2015, p. 166). Así, la presente investigación tiene relevancia como propuesta metodológica para analizar las diferencias socio-territoriales en el estado de Guerrero; debido a que el VIM es una técnica multivariada que facilita determinar las diferencias que existen entre unidades territoriales calificadas como muy homogéneas o bien las semejanzas presentes en aquellas unidades calificadas como muy heterogéneas, lo que resulta útil para clasificar un conjunto de demarcaciones a partir del índice construido con la información aportada por variables estadísticas, las cuales son seleccionadas según el marco conceptual de la investigación (García de León, 2006).

De esta manera, este trabajo tuvo como objetivo revelar las diferencias territoriales guerrerenses, con base en el tratamiento metodológico de indicadores socioeconómicos que han sido utilizados con tal finalidad, en trabajos precedentes sobre el territorio guerrerense. La hipótesis de trabajo gravitó en torno al escenario que se describe a continuación: en el estado de Guerrero, los municipios que poseen asentamientos urbanos con importancia turística, económico-comercial o político-administrativa suelen registrar mejores condiciones socioeconómicas. Estas ciudades funcionan como lugar central que enlaza distintas áreas rurales del municipio con la ciudad en cuestión.

\section{MARCO DE REFERENCIA}

En los últimos veinte años, en México se han concretado varios trabajos que exponen las desproporciones territoriales, presentes al interior de un estado, desde la óptica de la Asimilación Económica. Esta teoría tiene como objetivo fundamental revelar el grado de dominio que se tiene sobre un territorio mediante la evaluación de la intensidad de utilización de éste, el nivel de desarrollo de sus fuerzas productivas, la historia de poblamiento y otras particularidades del territorio abordado (Zailsev, 1972 citado por García, 1993). En ese orden de ideas, la intensidad del proceso de asimilación económica constituye la expresión medible determinada mediante el manejo metodológico de criterios seleccionados que se refieren a determinadas unidades espaciales (estados, municipios, localidades).

La aprensión cognoscitiva de la Asimilación Económica ha requerido del análisis regional integrado a la Tipificación Probabilística para revelar “... una imagen intelectual que facilita entender y explicar las 
similitudes y diferencias que acontecen entre distintos territorios a una escala previamente seleccionada" (Propin y Sánchez, 1998, p. 64). Dicha vertiente metodológica requiere que el número total de indicadores empleados no sea mayor a cinco, ni menor a tres. Éstos deben concebirse como mediciones complejas que revelan el comportamiento esencial del fenómeno de interés, lo cual supone poner mayor atención sobre la cualidad-complejidad de los atributos elegidos, por encima de la cantidad de indicadores seleccionados (Propin, 2003).

Con base en lo anterior, Propin y Sánchez (1998) optaron por los indicadores socioeconómicos siguientes: densidad de población, grado de urbanización, concentración regional de las tierras con riego, concentración espacial de la industria y densidad vial. Estos geógrafos argumentan que el primer indicador tiene trascendencia porque proporciona un panorama inicial de la asimilación económica que presenta un territorio, pues “... la presencia humana lleva implícita alguna actividad económica relacionada, en mayor o menor cuantía, con el medio natural donde se asienta" (Propin y Sánchez, 1998, p. 61). Por lo tanto, se interpreta que cuanto mayor sea la densidad demográfica, se torna más compleja la relación entre la sociedad y su entorno físico. Tal escenario adquiere mayor precisión cuando el dato se lee, de manera simultánea, con las cifras correspondientes a los cuatro indicadores socioeconómicos restantes.

El grado de urbanización “... precisa a las agrupaciones humanas como lugares donde se concentra una infraestructura social, típica del medio urbano; mientras mayor es el asentamiento poblacional o la concentración poblacional urbana en un espacio determinado, mayor será la posibilidad de ubicación de construcciones vinculadas con la infraestructura y los servicios sociales, y mayor es la atracción de la población procedente de otros territorios que carecen de la base social referida" (Propin y Sánchez, 1998, p. 61). En tanto, la densidad vial evidencia el grado de articulación que ostenta un territorio, aspecto considerado como un rasgo geográfico substancial para el proceso de asimilación económica; en la medida que “... los entes sociales y los medios de transformación de la naturaleza llegan a un territorio virgen por una vía de transportación previamente condicionada” (Propin y Sánchez, 1998, p. 62). 
El estado de Guerrero ha sido analizado, en varias ocasiones, con base en el marco conceptual y metodológico propuesto por la Teoría de la Asimilación Económica del Territorio. Al respecto, García (2011) señala que el análisis de la dinámica espacial guerrerense, así como la elaboración de mapas que la sintetice, es un aporte substancial a la elucidación del origen y las consecuencias de los desequilibrios territoriales de la entidad. Por su relevancia académica y relación directa con el propósito investigativo de este trabajo, a continuación se presentan algunas aportaciones hechas en tres de esas obras.

Propin y Sánchez (1998) sugieren que el estado de Guerrero posee disparidades socio-territoriales considerables; en la entidad, se determinaron múltiples estadios de asimilación económica derivados de la presencia de centros de primera importancia como Acapulco, tradicionales como Chilpancingo, Iguala y Taxco, y de reciente asimilación económica como Ixtapa-Zihuatanejo. A finales de los noventas, la densidad de población y la concentración de la industria básica constituían los indicadores primordiales para entender la diferenciación regional del estado, debido al bajo desarrollo urbano-industrial del mismo. Por el contrario, la densidad vial se manifestaba de manera irregular; sin aludir a la suficiencia de la red vial, ésta se presenta, indistintamente, con valores altos en los territorios menos asimilados.

Por su parte, Vázquez (2000) apunta que esta entidad mexicana posee abundantes recursos naturales y humanos aptos para su aprovechamiento; no obstante, al mismo tiempo, es una de las entidades federativas con el mayor grado de marginación socioeconómica del país. El impulso del gobierno federal, estatal y el capital privado, orientado al apoyo de actividades económicas específicas como el turismo, la minería, la agricultura y la industria en zonas determinadas de su territorio, el rezago de muchas otras, así como la contradicción entre los intereses generados en torno a espacios selectos para la economía y las de la población local, han dado origen al incremento de las disparidades socioeconómicas internas que se han acentuado en años recientes. Estas condiciones han dado lugar a la formación de regiones económicas disímiles que, acentuadas por la influencia de factores físico-geográficos, históricos, económicos, políticos y culturales, muestran la polarización territorial de la sociedad y la economía en Guerrero. 
En ese mismo tenor, García et al. (2015) proponen que la evolución geo-histórica acaecida sobre un entorno físico variado, ha dado un carácter especial y propio a Guerrero. Así lo permiten vislumbrar los contrastes socioeconómicos que prevalecen entre sus distintos municipios. De la región Norte a la Costa y de la Montaña a la Tierra Caliente, la entidad es biodiversa y cuenta con un vasto patrimonio históricoetnográfico-cultural que le confiere un lugar significativo dentro de la vida nacional. Sin embargo, sólo unos cuantos sitios, entre ellos Acapulco, Ixtapa-Zihuatanejo, Chilpancingo, Iguala y Taxco, detentan la mayor concentración de habitantes, inversiones económicas y actividades productivas rentables, sobre todo pertenecientes al sector terciario. Asimismo, estas demarcaciones albergan los núcleos urbanos más importantes de la entidad. En la mayoría de ellos, la expansión económico-demográfica reciente se debe a su actividad turística, su función político-administrativa o bien su papel notable en el abastecimiento regional de bienes y servicios.

\section{MATERIALES Y MÉTODO}

Esta investigación utiliza información estadística generada por el Instituto Nacional de Estadística y Geografía (INEGI). El manejo de esos datos oficiales se hizo con base en el método del Valor Índice Medio (VIM), éste fue construido con indicadores socioeconómicos cuya eficacia para reflejar disparidades territoriales ha sido confirmada en investigaciones previas sobre el estado de Guerrero; estos fueron: 1) densidad de población DP (muestra la relación entre el total de habitantes de un municipio y la extensión territorial de éste), 2) grado de urbanización GU (es un valor porcentual que evidencia la proporción de población que reside en asentamientos urbanos, sitios con 15000 o más personas (Unikel, 1978), respecto al número total de habitantes que registra cada municipio analizado), 3) concentración regional de las tierras con riego CTA (el valor correspondiente a este indicador revela el porcentaje de tierras con regadío, presentes en cada municipio, en relación con la superficie agrícola estatal de ese tipo), 4) concentración espacial de la industria CTI (es la cifra que resulta al dividir el valor de la producción de las ramas básicas del sector secundario entre la superficie del municipio en el que se desarrollan tales actividades económicas), y 5) densidad vial DV (la relación que existe entre la longitud de la red vial que ostenta cada municipio y el área del mismo). 
La investigación de García de León (2006) puntualiza como calcular el VIM, en un conjunto de unidades territoriales (estados, municipios, localidades). Los pasos medulares son: 1) estructurar una base de datos con los indicadores socioeconómicos para cada municipio del estado de Guerrero (Cuadro 1), 2) establecer la media aritmética y la desviación típica del conjunto de datos asociado con cada indicador (Cuadro 2), 3) tipificar los valores originales de la base de datos estructurada en el primer paso (se resta la media aritmética a los valores de los indicadores y el resultado se divide entre la desviación típica) (Cuadro 3), 5) calificar los valores tipificados con base en los rangos calculados para el conjunto de datos (Cuadro 4 y 5), 6) calcular el VIM (resulta del promedio de los cinco valores tipificados que caracterizan a cada municipio guerrerense) (Cuadro 6), y 6) calificar cualitativamente el valor índice medio con base en los rangos calculados para éste (Cuadro 7). Esta secuencia dio pauta para conocer cuál es la combinación específica de indicadores socioeconómicos que dan lugar a las condiciones socioeconómicas que diferencian a los municipios guerrerenses.

\begin{tabular}{|lccccc|}
\hline Municipio & DP & GU & CTI & CTA & DV \\
\hline Acapulco de Juárez & 470.051 & 83.077 & 559.840 & 382.030 & 0.291 \\
\hline Arcelia & 40.186 & 59.495 & 15.523 & 367.482 & 0.377 \\
\hline Atoyac de Álvarez & 41.921 & 35.453 & 38.778 & 427.250 & 0.344 \\
\hline Ayutla de los Libres & 66.641 & 20.612 & 16.318 & 300.775 & 0.588 \\
\hline Chilapa de Álvarez & 172.542 & 23.991 & 61.342 & 151.947 & 0.378 \\
\hline & ----- & ----- & ----- & ----- & ------ \\
\hline Zirándaro & ---- & ---- & ---- & ---- & ----- \\
\hline Zitlala & 8.508 & 0.000 & 1.490 & 202.537 & 0.208 \\
\hline Media aritmética & 75.395 & 0.000 & 26.978 & 169.322 & 0.414 \\
\hline Desviación típica & $\mathbf{6 0 . 3 5 5}$ & $\mathbf{1 1 . 0 3 1}$ & $\mathbf{4 0 . 5 6 6}$ & $\mathbf{2 5 1 . 6 8 1}$ & $\mathbf{0 . 3 3 7}$ \\
\hline
\end{tabular}

Cuadro 1. Ejemplo del comportamiento cuantitativo de los indicadores seleccionados. Fuente: elaborado con base en INEGI, 2019.

* Calculadas con base en el conjunto de datos de todos los municipios del estado de Guerrero. Nota: las líneas segmentas advierten la existencia de datos de los otros 72 municipios.

\begin{tabular}{|lccccc|}
\hline Municipio & DP & GU & CTI & CTA & DV \\
\hline Acapulco de Juárez & 6.159 & 3.240 & 5.304 & 0.519 & -0.302 \\
\hline Arcelia & -0.303 & 2.179 & -0.256 & 0.461 & 0.262 \\
\hline Atoyac de Álvarez & -0.277 & 1.098 & -0.018 & 0.699 & 0.045 \\
\hline Ayutla de los Libres & 0.094 & 0.431 & -0.248 & 0.195 & 1.643 \\
\hline Chilapa de Álvarez & 1.687 & 0.583 & 0.212 & -0.397 & 0.267 \\
\hline & ------ & ------ & ----- & ----- & ---- \\
\hline
\end{tabular}




\section{Castro e Peñaloza}

\begin{tabular}{|c|c|c|c|c|c|}
\hline & ------ & ------ & ------ & ------ & ------ \\
\hline Zirándaro & -0.779 & -0.496 & -0.399 & -0.196 & -0.851 \\
\hline Zitlala & 0.226 & -0.496 & -0.139 & -0.328 & 0.505 \\
\hline
\end{tabular}

Cuadro 2. Ejemplo de indicadores tipificados. Fuente: elaborado con base en el cuadro 1. Nota: las líneas segmentas advierten la existencia de datos de los otros 72 municipios.

\begin{tabular}{cl} 
Calificación & Rango de aplicación \\
\hline 1 & Menor a $-1,0$ \\
\hline 2 & Entre $-1,0$ y $-0,50$ \\
\hline 3 & Entre $-0,51$ y 0,50 \\
\hline 4 & Entre 0,51 y 1,0 \\
\hline 5 & Mayor a 1,0 \\
\hline
\end{tabular}

Cuadro 3. Calificaciones para valores tipificados.

Nota: 1 . Si el valor tipificado resultante es menor de -1,00, se le asigna calificación de 1 (la más baja de todas, debido a que corresponde a un valor original también muy bajo). 2 . Entre - 0,50 y -1,0, calificación de 2 (corresponde también a un valor original bajo, pero ya no tanto). 3. De - 0,50 a 0,50 va la calificación de 3 (Por un valor original ya más cercano a la media, donde la teoría de la probabilidad asume que se tendrán $68 \%$ del total de casos). 4 . De 0,5 a 1,0 califica con 4 . Se trata de un valor original que supera la media del conjunto. 5. Mayor de 1,0 obtendrá 5. El valor más alto de todos ya que supera por mucho la media (García de León, 2016).

\begin{tabular}{|lccccc|}
\hline Municipio & DP & GU & CTI & CTA & DV \\
\hline Acapulco de Juárez & 5 & 5 & 5 & 4 & 3 \\
\hline Arcelia & 3 & 5 & 3 & 3 & 3 \\
\hline Atoyac de Álvarez & 3 & 5 & 3 & 4 & 3 \\
\hline Ayutla de los Libres & 3 & 3 & 3 & 3 & 5 \\
\hline Chilapa de Álvarez & 5 & 4 & 3 & 3 & 3 \\
\hline & ------ & ----- & ----- & ----- & ----- \\
\hline Zirándaro & ----- & --------- & 2 \\
\hline Zitlala & 2 & 3 & ------ & ----- & -1 \\
\hline
\end{tabular}

Cuadro 4. Ejemplo de la cualificación de los indicadores utilizados. Fuente: elaborado con base en el cuadro 2 y 3.

Nota: las líneas segmentas advierten la existencia de datos de los otros 72 municipios.

\begin{tabular}{|lcccccc|}
\hline Municipio & DP & GU & CTI & CTA & DV & VIM \\
\hline Acapulco de Juárez & 5 & 5 & 5 & 4 & 3 & 4.4 \\
\hline Arcelia & 3 & 5 & 3 & 3 & 3 & 3.4 \\
\hline Atoyac de Álvarez & 3 & 5 & 3 & 4 & 3 & 3.6 \\
\hline Ayutla de los Libres & 3 & 3 & 3 & 3 & 5 & 3.4 \\
\hline Chilapa de Álvarez & 5 & 4 & 3 & 3 & 3 & 3.6 \\
\hline & ----- & ----- & ------- & ----- & ---- \\
\hline Zirándaro & ------ & ----- & ------ & ----- & 2 & 2.6 \\
\hline Zitlala & 2 & 3 & 3 & 3 & 3 & 3.2 \\
\hline
\end{tabular}

Cuadro 5. Ejemplo del cálculo del VIM. Fuente: elaborado con base en el cuadro 4.

Nota: las líneas segmentas advierten la existencia de datos de los otros 72 municipios. 


\begin{tabular}{|ccc|}
\hline Menor a 2.64 & 1 & Muy baja \\
\hline 2.65 a 3.08 & 2 & Baja \\
\hline 3.09 a 3.52 & 3 & Media \\
\hline 3.53 a 3.96 & 4 & Alta \\
\hline Mayor a 3.96 & 5 & Muy alta \\
\hline
\end{tabular}

Cuadro 6. Calificaciones para el VIM.

Nota: con el fin de ordenar los municipios en rangos de clasificación adecuados, se aprovechó el valor del índice medio obtenido. Con base en los valores obtenidos, se observó la posibilidad de formar cinco grandes grupos "naturales". A partir de esa agrupación es posible jerarquizar los municipios, en rangos con amplitud aceptable, justificados por su similitud con la secuencia que presentan las unidades tipificadas que permiten formar la curva de distribución normal (García De León, 2006).

\begin{tabular}{|lcc|}
\hline Municipio & VIM & Condición \\
\hline Acapulco de Juárez & 4.4 & 5 \\
\hline Arcelia & 3.4 & 3 \\
\hline Atoyac de Álvarez & 3.6 & 4 \\
\hline Ayutla de los Libres & 3.4 & 3 \\
\hline Chilapa de Álvarez & 3.6 & 4 \\
\hline & ----- & ----- \\
\hline Zirándaro & ----- & ----- \\
\hline Zitlala & 2.6 & 1 \\
\hline
\end{tabular}

Cuadro 7. Ejemplo de indicadores tipificados. Fuente: elaborado con base en el cuadro 1.

Nota: las líneas segmentas advierten la existencia de datos de los otros 72 municipios.

\section{ÁREA DE ESTUDIO}

El estado de Guerrero se ubica al sur de la República Mexicana. Esta entidad federativa cuenta con una extensión territorial de 63794 km2 (3.2\% de la superficie del país). La división político-administrativa guerrerense está conformada por ochenta y un municipios, los cuales se agrupan en las regiones siguientes: Acapulco, Centro, Costa Grande, Costa Chica, Montaña, Norte y Tierra Caliente (Cuadro 8, Figura 1). De acuerdo con el INEGI, Guerrero tiene 3533251 habitantes (3.0\% del total nacional). Por el número de habitantes destacan las demarcaciones siguientes: Acapulco de Juárez (810 669), Chilpancingo de los Bravo (273 106), Iguala de la Independencia (151 660), Chilapa de Álvarez (129 867), Zihuatanejo de Azueta (124 824) y Taxco de Alarcón (108 416). En Guerrero, 78\% de la población reside en asentamientos urbanos y 22\% en localidades rurales (INEGI, 2019) (Figura 2).

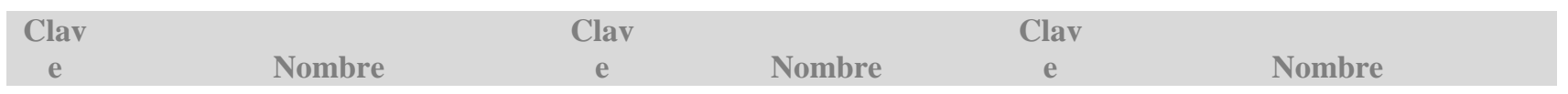




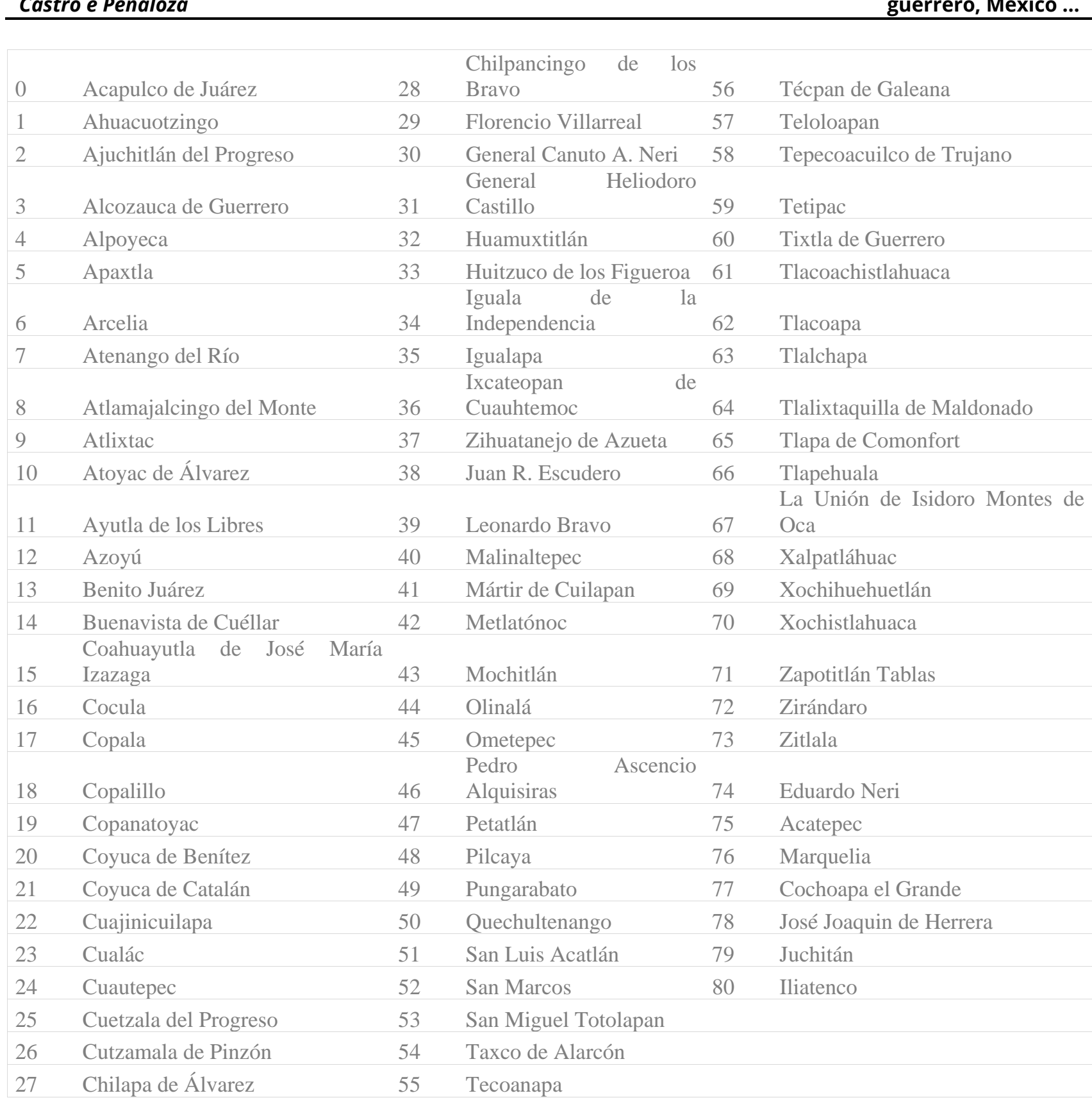

Cuadro 8. Espesor en centímetros por caída de ceniza en las comunidades La Pastora y El Tapojo según escenarios de Soto (2012). Fuente: Soto (2012). 


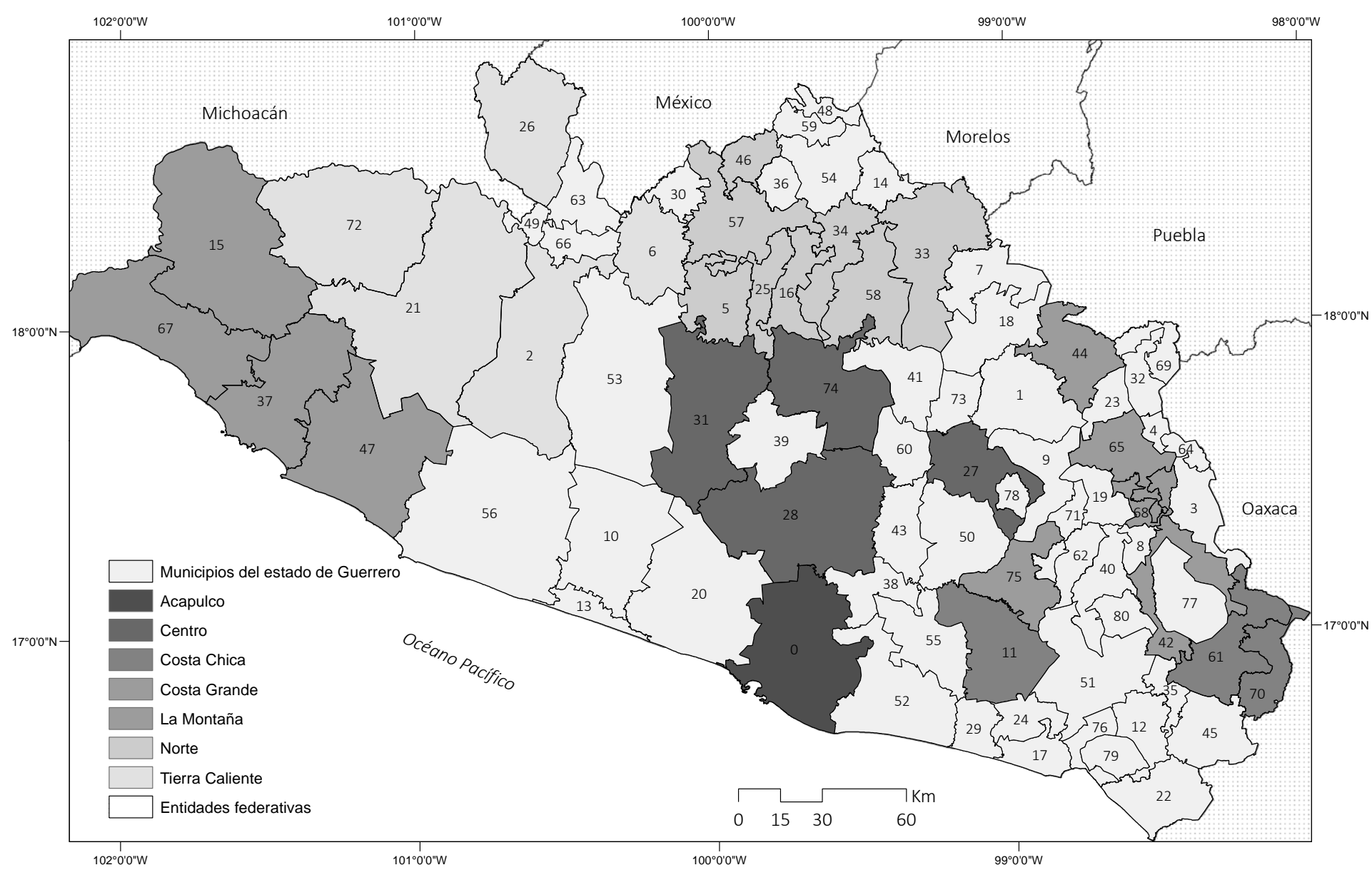

Figura 1. Guerrero: división político-administrativa

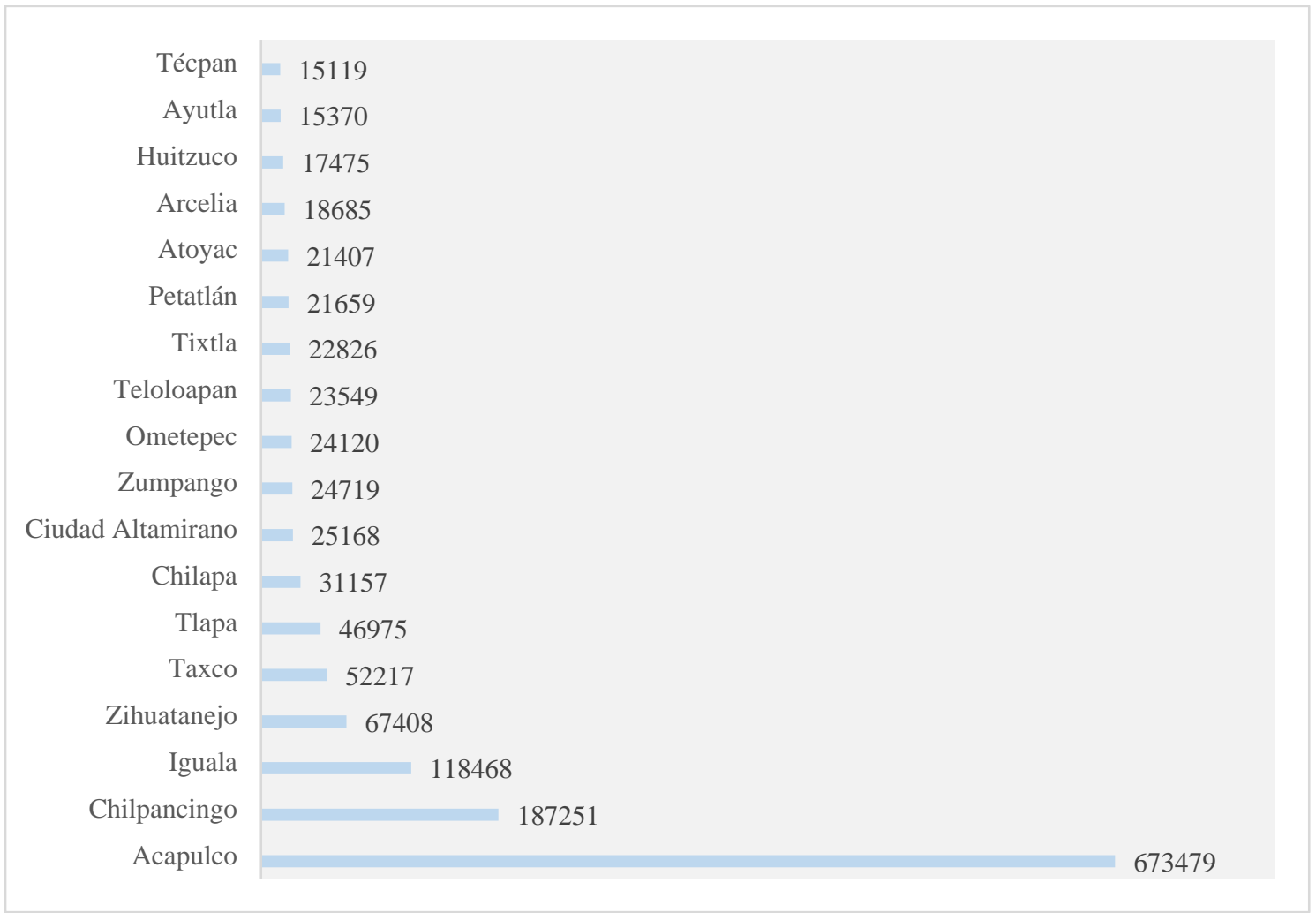

Figura 2. Guerrero: principales ciudades, según su número de habitantes. Fuente: elaborado con base en INEGI, 2019. 
Esta entidad mexicana cuenta con una Población Económicamente Activa de 1421962 . El sector económico con más activos fue el terciario, en total 668047 personas, esto es 59.2\% de la población ocupada de la entidad. Por su parte, el primario y el secundario reportaron 20.9 y $18.4 \%$, respectivamente. Por la cantidad de Población Económicamente Activa (PEA), destacaron los municipios siguientes: Acapulco de Juárez, 642 133; Chilpancingo de los Bravo, 210 230; Iguala de la Independencia, 117 480; Zihuatanejo de Azueta, 97 907; Chilapa de Álvarez, 92 841; Taxco de Alarcón, 85736; Tlapa de Comonfort, 62666 y Coyuca de Benítez, 58355 (INEGI, 2019). Asimismo, cabe destacar que el sector terciario aporta 76\% del Producto Interno Bruto Estatal (PIBE); las actividades secundarias, $18.4 \%$; y el sector primario, $5.6 \%$.

En los últimos decenios, el turismo ha sido el principal eje de la dinámica económica de Guerrero; tal actividad se aglutina en asentamientos humanos de la costa como Acapulco, Ixtapa y Zihuatanejo, y en la ciudad de Taxco. La mayor parte del territorio guerrerense ha permanecido al margen de la derrama económica que genera esta actividad terciaria. En el estado, la modernización del sector primario y secundario no ha tenido lugar por la falta de inversiones públicas y privadas, a pesar de que la entidad cuenta con diversas áreas con características tanto físicas como socioeconómicas que podrían favorecer la creación de proyectos productivos y, por lo tanto, atenuar la falta de alternativas laborales y los problemas sociales asociados con ella (García et al., 2016).

De acuerdo con el Consejo Nacional de Evaluación de la Política de Desarrollo Social (CONEVAL), en Guerrero hay 825000 personas en situación de pobreza extrema, esto es 23\% de los guerrerenses. En la entidad, $73.9 \%$ de los habitantes tiene carencias vinculadas con el acceso a la seguridad social; 50.2\%, asociadas con los servicios básicos en sus viviendas; $31.7 \%$, relacionadas con la calidad y dimensiones de la vivienda; $27.2 \%$, ligadas con el acceso a la alimentación; $25.2 \%$ presenta rezago educativo y $13.3 \%$ muestra deficiencias en el acceso a los servicios de salud (CONEVAL, 2018). El rezago socioeconómico ha sido persistente, en la mayoría de los municipios, esta situación se ha agudizado en la región Centro, La Montaña y en la Costa Chica, territorios caracterizados por la presencia ancestral de etnias indígenas mixtecas, amuzgas, tlapanecas y nahuas. Por lo general, en estos sitios aún prevalecen localidades 
dispersas, carentes de infraestructura básica y escasamente articuladas, cuyo principal medio de subsistencia es el desempeño de actividades primarias.

En ese contexto, un número importante de guerrerenses emigra a otras entidades federativas o a Estados Unidos. Con respecto al primer tipo de migración, la población se desplaza a estados como Morelos, México, Baja California, Ciudad de México y Michoacán. De acuerdo con el Centro de Derechos Humanos Tlachinollan, en 2015, durante el lapso comprendido entre septiembre y enero, 8200 campesinos guerrerenses se trasladaron a los campos agrícolas de otras entidades mexicanas; 73\% viajó a Sinaloa, $13 \%$ a Baja California y 5\%, a Sonora y Chihuahua, respectivamente. Por otra parte, el estado de Guerrero, junto con Michoacán y Oaxaca, son las entidades con mayor porcentaje de población que emigra a Estados Unidos; en Guerrero, 89\% de la población migrante internacional se desplaza a ese país. Por lo general, se trasladan a Oregón, California, Arizona, Mississippi, Florida, Nueva York, Virginia o Carolina del Norte.

En La Montaña, la parte serrana de la Costa Chica y la Sierra, las personas que se resisten a tal desarraigo o que no pueden hacerlo; además de la pobreza tienen que habituarse a las operaciones del Ejército Mexicano; ya que tanto para el gobierno federal como para el estatal, esta porción del territorio guerrerense, constituyen un foco rojo debido a la presencia de áreas en las que se suele cultivar marihuana y amapola. Además, en el último decenio, en las siete regiones de la entidad se ha registrado la presencia de grupos de la delincuencia organizada que se disputan el control de distintos segmentos de Guerrero. De acuerdo con la Secretaría de la Defensa Nacional (SEDENA), la actividad criminal se concentra en 81 municipios del estado, destacan las jurisdicciones comprendidas en los corredores Acapulco-Chilpancingo-Chilapa; Costa Grande (desde Coyuca de Benítez a La Unión) y Tierra CalienteNorte.

A esto hay que sumar que, en algunos años más, municipios como San Luis Acatlán, Metlatónoc, Tlacoapa, Atlamajalcingo del Monte, Malinaltepec, Acatepec y Zapotitlán Tablas podrían registrar el arribo de compañías mineras, un acontecimiento que detonará la contaminación y destrucción del entorno físico de La Montaña y la porción serrana de la Costa Chica, así como distintos conflictos socioterritoriales debido a la expropiación de varios ejidos de esas regiones. Por lo tanto, en los próximos 
años, de nuevo, tendrá lugar un crecimiento económico y demográfico en unos cuantos sitios del estado, mientras que segmentos amplios de él permanecerán al margen de tal dinámica. Las situaciones descritas evidencian la complejidad de la estructura económico-territorial del estado de Guerrero.

\section{Diferencias territoriales del estado de Guerrero desde la perspectiva del VIM}

Con base en la metodología del Valor Índice Medio, el estado de Guerrero presenta las condiciones socioeconómicas siguientes (Figura 3):

Muy alta. Corresponde a los municipios de Acapulco de Juárez, Iguala de la Independencia, Ometepec, Pungarabato y Taxco de Alarcón. Estas cinco circunscripciones concentran 1175880 habitantes (33.3\% del total de guerrerenses). Las cabeceras de estas demarcaciones superan los 15000 habitantes; destacan, las ciudades de Acapulco, con 673479 personas; Iguala, con 118468 personas; y Taxco, con 52217 personas (INEGI, 2019). Otros rasgos socioeconómicos sobresalientes de este conjunto son: participan con 56.7\% del valor de la producción manufacturera, 10.1\% del valor de la producción agrícola y 6.4\% de la infraestructura carretera (INEGI, 2019). Los municipios con condición muy alta presentaron un valor índice medio comprendido entre 4.0 y 4.2 puntos; como resultado del predominio de valores muy altos (5); en la mayor parte de los indicadores ponderados; sobre todo la población relativa (DP), el grado de urbanización (GU) y la concentración de la producción industrial (CPI). Por otra parte, tanto la concentración de la producción agrícola (CTA) como la densidad vial (DV) son menos relevantes, en términos cuantitativos. 


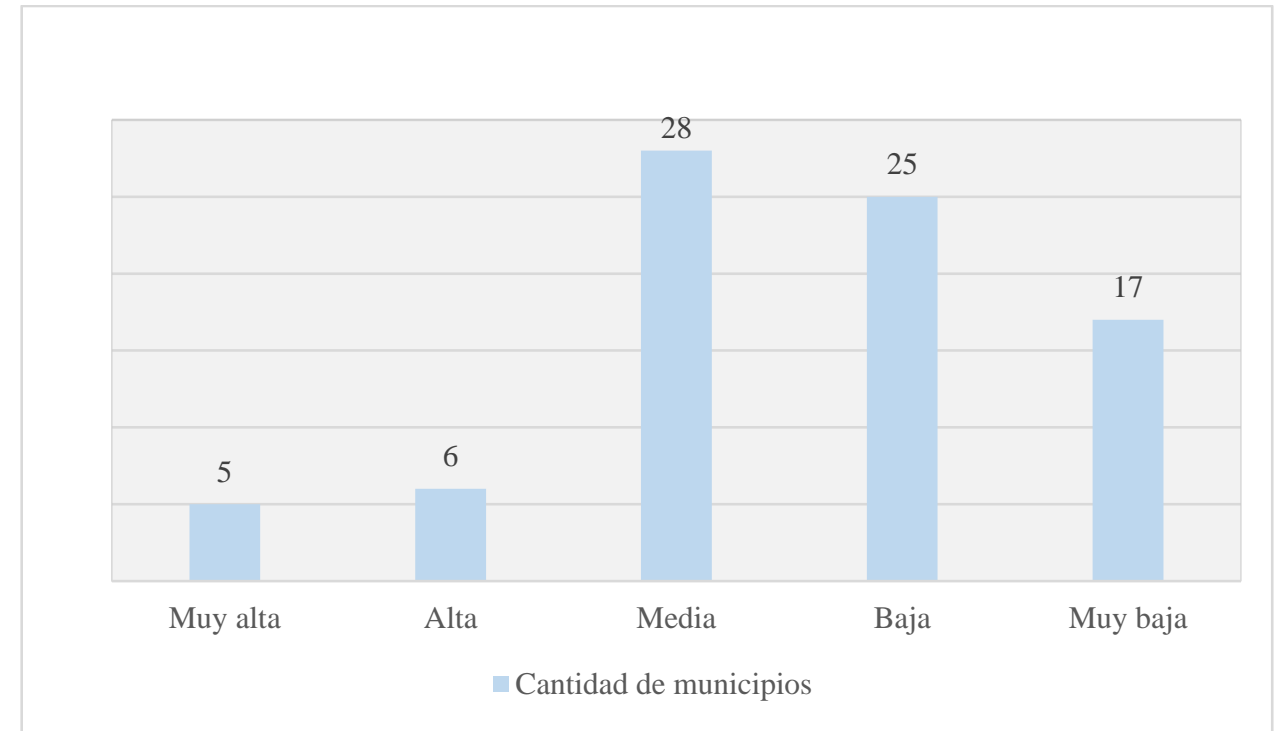

Figura 3. Guerrero: condiciones socioeconómicas a nivel municipal. Fuente: INEGI, 2019.

Alta. Se presentó en Atoyac de Álvarez, Chilapa de Álvarez, Cuautepec, José Joaquín de Herrera, Pilcaya y Tlapa de Comonfort. La población de estos seis municipios representa $9.2 \%$ de total de guerrerenses, esto es 325192 personas (INEGI, 2019). Únicamente Átoyac de Álvarez, Chilpa de Álvarez y Tlapa de Comonfort tienen cabeceras con 15000 o más personas. En estas demarcaciones se concentra 5.3\% de la producción manufacturera y 7.2\% de la producción agrícola. En términos de la infraestructura carretera, estos municipios cuentan con $7.5 \%$ de las vías de comunicaciones que existen en la entidad (INEGI, 2019). Por otra parte, en todos los municipios en condición alta, el valor índice medio fue de 3.6 puntos como resultado del predominio de montos significativos, altos (4) y muy altos (5) (Figura 4). 


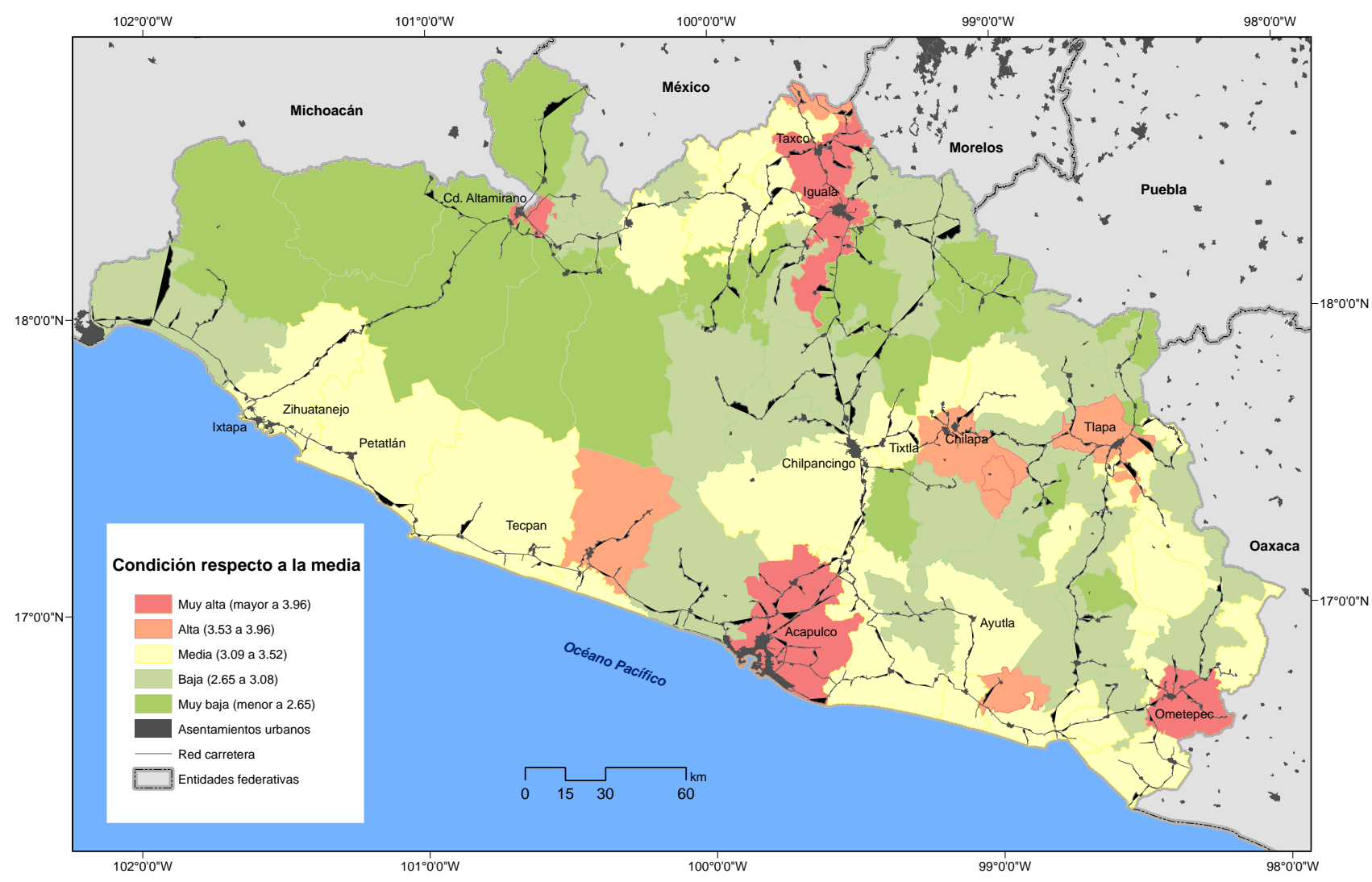

Figura 4. Guerrero: condición socioeconómica a nivel municipal. Fuente: INEGI, 2019.

Media. Se detectó en veintiocho municipios (35\% del total); un número considerable de éstos se localizan en la parte norte y en la costa. En conjunto reúnen 1065631 personas (30.2\% de la población estatal). Existen ocho asentamientos urbanos, las cabeceras de Chilpancingo de los Bravo, Zihuatanejo de Azueta, Teloloapan, Tixtla de Guerrero, Petatlán, Arcelia, Técpan de Galeana, Ayutla de los Libres. Este grupo de municipios tiene las características siguientes: $37 \%$ de la infraestructura carretera del estado, 26\% del valor de la producción manufacturera y 45.3\% del valor de la producción agrícola (INEGI, 2019). En estas unidades territoriales, el valor índice medio oscila entre 3.2 y 3.4 puntos como resultado del predominio valores no mayores a 3 puntos; en particular, la densidad demográfica, el grado de urbanización y la concentración territorial de la industria; ya que la concentración territorial agrícola y la densidad vial presentan valores notables.

Baja. Corresponde a un total de veinticinco municipios (31\% del conjunto analizado). La condición baja es notable en La Montaña, la parte serrana de la Costa Chica y occidente de la región Centro. En estas 
demarcaciones viven 668497 personas (19\% de los guerrerenses). Únicamente dos municipios tienen asentamientos con más de 15000 habitantes; estas son las cabeceras de Eduardo Neri (24 719) y Huitzuco de los Figueroa (17 475). Otro dato relevante es que en conjunto reúnen sólo 29\% de la infraestructura carretera estatal. Asimismo, este grupo de municipios participa con $9.1 \%$ del valor de la producción manufacturera de la entidad y 23.8\% del valor de la producción agrícola (INEGI, 2019). En este orden de ideas, en estas jurisdicciones el valor índice medio fluctuó entre 2.8 y 3.0 puntos; los indicadores evaluados suelen presentar valores por debajo de la media estatal; sobre todo, la concentración regional de la producción agrícola y la densidad vial.

Muy baja. Esta condición se identificó en diecisiete municipios de la entidad (21\% del total); la mayoría localizados en la parte norte y noroeste de Guerrero. Estos municipios concentran 298051 habitantes (8.4\% del total estatal). Los asentamientos humanos no rebasan los 15000 habitantes. En conjunto, aportan $3.1 \%$ del valor de la producción manufacturera y 13.6\% del valor de la producción agrícola (INEGI, 2019). Cuentan con 21\% de la red carretera. En esas demarcaciones, el valor índice medio estuvo comprendido entre 2.2 y 2.6 puntos; como resultado del predominio de valores no significativos en los cinco indicadores ponderados. En particular, los valores relacionados con la densidad vial, la población relativa y la concentración territorial de la producción agrícola; los tres estuvieron por debajo, de manera importante, del promedio estatal. En contraste, el grado de urbanización y la concentración territorial de la producción industrial presentó 3 puntos.

Las condiciones socioeconómicas por debajo de la media estatal predominan en aquellas áreas dónde la principal fuente de ingresos de la población es la agricultura con rendimientos bajos. Al igual que en la mayoría de los espacios rurales de México, esa actividad económica se desarrolla en parcelas pequeñas y con inversiones económicas mínimas que no dan pauta para el uso significativo de técnicas modernas de cultivo. Otra limitante es la presencia de áreas extensas de relieve escarpado, la cual también ha dificultado el trazo de vías de comunicación, indispensables para la integración socioeconómica regional.

En contraste, los municipios que poseen asentamientos urbanos con importancia turística, económicocomercial o político-administrativa suelen registrar mejores condiciones socioeconómicas. Estas ciudades funcionan como lugar central que enlaza distintas áreas rurales de la demarcación con la ciudad en 
cuestión. El aislamiento de numerosos asentamientos en Guerrero, originado por el relieve escarpado y la escasa infraestructura carretera existente en la entidad, ha dado pauta al fortalecimiento de ciudades pequeñas que operan como capitales subregionales, tal es el caso de algunas cabeceras municipales guerrerenses. La función y relevancia de esos sitios es más significativa cuanto mayor es la distancia que los separa de los principales centros urbanos; eso les dota una condición estratégica para la estructuración territorial, con efectos dinamizadores sobre los espacios adyacentes (Pumain, 1997 citado por Capel, 2009).

En la región Centro, sobresalen las ciudades de Chilpancingo, Chilapa y Tixtla. La influencia socioeconómica de la primera se extiende, básicamente, sobre demarcaciones de la porción centro-oriente de la entidad y del centro de la Costa Chica. Esta ciudad debe su relevancia regional a la función políticoadministrativa como capital de la entidad, la cabecera municipal es la segunda con más guerrerenses y es atravesada por la carretera México-Acapulco (eje principal de comunicación de la entidad), una característica que dio pauta para fortalecerse como el sitio que proporciona suministros y servicios importantes a quienes habitan en las demarcaciones circundantes, así como a las personas que se desplazan por la vía de comunicación señalada o bien desde la Costa Chica hacia el centro del país.

Por otra parte, Chilapa tiene un vínculo socioeconómico substancial con Zitlala, Ahuacuotzingo, Atixtlac, José Joaquín Herrera y Quechultenango, ya que esta ciudad cuenta con mayor gama de comercios y servicios. Por lo general, los domingos hay más afluencia de compradores y vendedores porque se instala el tianguis, de origen prehispánico, más grande de la entidad. Tixtla está a $15 \mathrm{~km}$ de Chilpancingo y constituye el paso obligado para la población que se desplaza hacia el centro-oriente de Guerrero (principalmente a La Montaña) y de esa zona a la capital. Con frecuencia, esta última es abastecida de productos agrícolas que se cultivan en el valle de Tixtla. Además, ésta posee ciertos recursos de interés para la economía turística que, aunados a su proximidad al principal eje de comunicación de la entidad, han favorecido el arribo de visitantes.

En La Montaña, la poca infraestructura carretera de la región converge en la ciudad de Tlapa de Comonfort, allí ha crecido la cantidad de comercios y servicios que se oferta. Esto es producto de la 
expansión demográfica de la misma y del número importante de remesas, procedentes de Estados Unidos, que capta. Si bien aún es más frecuente que familias nahuas, tlapanecas y mixtecas se desplacen al noroeste de México para trabajar como jornaleros agrícolas; en los dos últimos decenios, creció la migración, en especial de población masculina, hacia ciudades como Nueva York o Chicago. Incluso, organizaciones como el Centro de Derechos Humanos de La Montaña Tlachinollan expresan que esta última urbe norteamericana es el asentamiento con más guerrerenses después de Acapulco.

En la Costa Chica son notables las ciudades de Ometepec y Ayutla; la primera es fundamental para la dinámica económica del oriente de la Costa Chica, pues es la localidad que tiene intercambio comercial constante con Igualapa, Tlacoachistlahuaca, Xochistlahuaca, Azoyú, Marquelia y Cuajinicuilapa (García, 2011). En los tres primeros predomina la población indígena amuzga, mixteca y nahua, y afrodescendientes, en las demás circunscripciones. Por lo general, esta población sólo cuenta con recursos económicos modestos, apenas suficientes para desplazarse hasta la cabecera de Ometepec para vender sus productos agrícolas o artesanías, y adquirir productos que no encuentran en su demarcación o bien recibir atención médica especializada, porque allí hay un hospital regional. La presencia de éste acentúa el carácter estratégico de la ciudad, debido a que su área de atracción se expande al centro y oriente de la Costa Chica.

La ciudad de Ayutla es el segundo asentamiento más poblado de la región. Abastece diversos productos y servicios a los pueblos de las circunscripciones contiguas. Desde hace varios decenios, ha sostenido un intercambio comercial importante con los municipios de Acatepec, San Luis Acatlán, Cuautepec, Florencio Villareal y Tecoanapa, aunque su influencia suele extenderse hasta jurisdicciones del suroeste de La Montaña, debido a que muchos de éstos no cuentan con servicios básicos como unidades de atención médica, instituciones educativas de bachillerato o de nivel superior. Además, en la localidad de Ayutla es relevante la presencia de acaparadores de la producción agrícola regional, quienes se encargan de transportarla y venderla en las ciudades de Acapulco, Chilpancingo y México. La actividad comercial municipal hace uso de los tramos de carretera federal que lo comunica con la capital de la entidad. Esto último es estratégico para los habitantes del centro-oriente de la Costa Chica que desean desplazarse a Chilpancingo o a la Ciudad de México, ya que, en la entidad, es la ruta más corta. 
En la Costa Grande, Ixtapa-Zihuatanejo es el asentamiento humano con mayor importancia socioeconómica. Ixtapa-Zihuatanejo es el segundo destino vacacional guerrerense de mayor trascendencia económica. Este centro vacacional comenzó a erigirse en el decenio de los setentas, los planes oficiales de desarrollo turístico del país tenían contemplado que el surgimiento y consolidación de este tipo de polo turístico propiciaría el desarrollo socioeconómico de las zonas adyacentes. Sin embargo, la falta de inversiones substanciales en esas áreas y otras de la entidad ha ampliado las disparidades regionales previamente existentes. Con más de cuatro decenios de antigüedad, Ixtapa-Zihuatanejo es uno de los pocos proyectos de Guerrero a los que se asigna más recursos económicos, tanto por el Estado como por la iniciativa privada. Este destino turístico se ha posicionado en los mercados de Estados Unidos y Canadá. Cada año, arriban visitantes procedentes, principalmente, de Houston, Los Ángeles, Phoenix, San Francisco, Seattle, Portland, San Antonio, Austin, Dallas, Chicago y Vancouver.

Por su parte, en la Tierra Caliente, Ciudad Altamirano, Arcelia y Teloloapan son los municipios que destacan en el contexto regional. Ciudad Altamirano, la cabecera de Pungarabato, es la localidad con más relevancia económico-comercial calentana. La configuración de la infraestructura de comunicaciones denota esa característica, pues las principales carreteras la conectan, directamente, con Cutzamala, Tlachapa, Tlapehuala y Coyuca de Catalán (cuatro de las nueve circunscripciones que integran a la región). Asimismo, desde el decenio de los cincuentas, la población de varias localidades michoacanas que colindan con Pungarabato, entre ellas Tiquicheo, Huetamo, San Lucas, Quetzería y Churumusco de Morelos, acude allí para realizar sus compras, ya que no cuenta con otra alternativa más cercana. Asimismo, Arcelia y Teloloapan fungen como centros de comercio intrarregional, la cabecera de Arcelia interactúa, en especial, con distintos poblados de General Canuto A. Neri, Tlalchapa, Tlapehuala y Ajuchitlán de Progreso. En tanto, la ciudad de Teloloapan con Ixcateopan, Cuetzala del Progreso, Apaxtla, General Canuto A. Neri y Pedro Ascencio Alquisiras. Además, debido a que ambos municipios colindan con el estado de México, su área de influencia se extiende hasta algunos poblados mexiquenses.

En la región Norte tienen un papel notable las ciudades de Iguala y Taxco. Iguala es el tercer centro urbano más importante de la entidad y forma parte del segmento septentrional con mayor relevancia 
económica. Esa ciudad ha sido un nodo económico-comercial sobresaliente con influencia sobre varias demarcaciones de la región Norte y de la Tierra Caliente; ha desempeñado esa función desde el período de dominio español. En esa época, su ubicación estratégica, sobre la única ruta que ligaba al puerto de Acapulco con el centro del país, favoreció la proliferación de comerciantes que acopiaban la producción agrícola de la costa y la Tierra Caliente para, posteriormente, venderla a otras áreas del territorio nacional. Al finalizar el siglo XIX, esa actividad económica se fortaleció, pues el ferrocarril vinculó a la localidad con otras entidades. Todavía, en 1980, Iguala era el segundo asentamiento más poblado, pero, gradualmente, la capital estatal lo desplazó de esa posición. En ese sentido, a principios del decenio de los noventa, la construcción de la Autopista del Sol causó cierta disminución de su relevancia económicoregional, ya que esa vía, la principal del estado, no atravesó al municipio. En tanto, la ciudad de Taxco no sólo cubre las necesidades de su municipio, sino también las de los habitantes de áreas vecinas; en especial, de localidades adyacentes de Tetipac, Pedro Ascencio Alquisiras, Ixcateopan y Pilcaya.

Acapulco es la ciudad con mayor importancia económica y demográfica de Guerrero. Desde el decenio de los cincuentas, la actividad turística ha sido estimulada por el gobierno mexicano; asimismo, más tarde, ya con el modelo neoliberal, también cobró importancia la inversión extranjera directa relacionada con esta actividad económica. Esto permitió que Acapulco se posicionará como uno de los principales destinos turísticos del país. Sin embargo, también trajo consigo la imposición de un conjunto de condiciones políticas y económicas que sólo garantizan la obtención de utilidades a los inversionistas. En consecuencia, se registraron impactos socio-ambientales diversos, sobre todo, en las áreas involucradas, directamente, con la puesta en marcha de proyectos turísticos. La mayoría de los conflictos, ha tenido lugar tras la disputa por espacios dotados de características físicas estratégicas para el desarrollo de los mismos, así como por la incertidumbre socioeconómica de las personas que laboran en este destino de playa; en particular aquellos con instrucción formal mínima, lo que los obliga a desempeñar trabajos informales, sin recibir seguridad social o tener certeza laboral.

\section{CONSIDERACIONES FINALES}


El tratamiento metodológico de indicadores socioeconómicos, con base en el Valor Índice Medio (VIM), ratifica algunos de los escenarios socio-territoriales definidos por especialistas en la Teoría de la Asimilación Económica del Territorio; destacan los siguientes: las condiciones socioeconómicas de los municipios guerrerenses advierten la intensidad de las inversiones económicas acaecidas con la finalidad de incentivar la práctica de actividades económicas específicas, tales es el caso del turismo, el comercio o los servicios. En ese contexto, es importante el papel que desempeñan los municipios de Acapulco, Chilpancingo, Iguala, Zihuatanejo de Azueta y Taxco; en particular las cabeceras municipales de estas demarcaciones son las ciudades guerrerenses que fungen como nodos económicos, sociales y políticos articulados con la dimensión macrorregional, nacional e incluso internacional. No obstante, es conveniente aclarar que aquellos municipios que registran condiciones socioeconómicas altas o muy altas no sugieren un supuesto patrón óptimo de desarrollo, sino únicamente la presencia de mayor número de habitantes, actividades productivas e infraestructura de comunicaciones, respecto al resto de los municipios de Guerrero.

De esta manera, las circunscripciones guerrerenses con condiciones socioeconómicas por debajo de la media estatal no siempre se asocian con aquellos espacios marginales y cuyas dificultades sociales han sido una constante a lo largo de la historia económico-productiva de la entidad; por ejemplo, los municipios de La Montaña o aquellas jurisdicciones con cantidad importante de población indígena de la región Centro y la Costa Chica, los cuales son espacios subdesarrollados que, independientemente de la condición socioeconómica detectada mediante el VIM, tienen un carácter vulnerable a situaciones coyunturales exógenas; y podrían estar sujetos a las decisiones sustentadas en el interés y voluntad del gran capital, sobre todo relacionado con la minería en los próximos años. A diferencia de los trabajos geográfico-económicos previos sobre el estado de Guerrero, esta investigación revelo que existe una cantidad notable de municipios con condiciones socioeconómicas bajas y muy bajas en las regiones Norte y Tierra Caliente, una situación que podría deberse al incremento de la actividad del crimen organizado ocurrida en el último decenio en ambas regiones.

Lo expuesto en este último párrafo robustece las nociones básicas que se tienen en torno a la Asimilación Económica del Territorio; cobra notabilidad el hecho de que la gradual incorporación de distintos espacios 
a la vida económica del territorio al que pertenece requiere el entendimiento del momento en que tiene lugar, las relaciones sociales en las que se inscribe y las características físicas del espacio en cuestión. Esto da pauta para identificar procesos simultáneos de asimilación económica en un territorio, debido a los diferentes estadios de desarrollo de las fuerzas productivas que se interactúan en él. Y por otra parte, también permite el reconocimiento de aquellos procesos de abandono de algunas demarcaciones ya asimiladas; debido, generalmente, al agotamiento, destrucción o pérdida del valor de sus recursos, como podría estar ocurriendo en varios municipios de la Tierra Caliente y la región Norte. Por tal motivo, como se ha señalado en la literatura especializada, el conocimiento holístico sobre las disparidades socioeconómicas de la entidad debe tomar en cuenta aquellos momentos relacionados con la adhesión de un territorio a determinada dinámica económica o social; tal es el caso del incremento de la actividad del crimen organizado o la inserción de la mineras extranjeras en Guerrero.

\section{REFERENCIAS}

Capel, H. (2009). "Las pequeñas ciudades en la urbanización generalizada y ante la crisis global". Investigaciones Geográficas. Boletín núm. 70. Instituto de Geografía, Universidad Nacional Autónoma de México. México. (pp. 7-32).

CONEVAL (2018). Medición de la pobreza 2010-2016. https://coneval.org.mx/Medicion/MP/Paginas/Pobreza_2016.aspx

García, A. (1993) "Asimilación económica del territorio (un nuevo enfoque en la interpretación regional del país)". Investigaciones Geográficas. Boletín núm. 27. Instituto de Geografía, UNAM. México. (pp. 69-94).

García de León, Armando (2015). Interacciones económicas transfronterizas y sus efectos en espacios nacionales. https://www.researchgate.net/publication/294572996_Teoria_y_metodos_de_la_Geografia_Cuantitativa_Libro_1_por_una _Geografia_de_lo_real (23-02-2016).

García De León, Armando (2006). Yucatán 1990-2000: evaluación del bienestar municipal y Regional a partir de las condiciones fisicas de las Viviendas. http://www.eumed.net/jirr/1/AMECIDER2006/PARTE\%201/70\%20Armando\%20Garcia\%20de\%20Leon\%20Loza.pdf (23-02-2016).

García, N. (2011). Los grados de asimilación económica del estado de Guerrero, a fines del siglo XX. Tesis de Doctorado en Geografía. Facultad de Filosofía y Letras, UNAM. México.

García, N., Villerías, S. y P. V. Tello (2015). "Niveles de asimilación económica del estado de Guerrero". RICSH Revista Iberoamericana de las Ciencias Sociales y Humanisticas, vol. 5, núm. 10, julio-diciembre. Centro de Estudios e Investigaciones para el Desarrollo Docente A.C.

INEGI (2019). Sistema Estatal y Municipal de Base de Datos. Recuperado de http://sc.inegi.org.mx/cobdem/

Propin, E. (2003). Teorias y Métodos en Geografía Económica. Instituto de Geografía, UNAM. México.

Propin, E. y Á. Sánchez (1998). "Niveles de asimilación económica del estado de Guerrero". Investigaciones Geográficas. Boletín núm. 37. Instituto de Geografía, UNAM. México. (pp. 59-70). 
Sánchez, A. (2000). Marginación e ingreso en los municipios de México: análisis para la asignación de recursos fiscales. México D.F.: Miguel Ángel Porrúa-Instituto de Investigaciones Económicas, UNAM.

Unikel, L. (1978). El desarrollo urbano de México: Diagnóstico e implicaciones futuras. 2da. Edición. El Colegio de México. México.

Vázquez, V. (2000). La regionalización económica del estado de Guerrero. Tesis de Maestría en Geografía. Facultad de Filosofía y Letras, UNAM. México. 